\title{
ON THE CLASS OF BANACH SPACES WITH JAMES CONSTANT $\sqrt{2}$, III
}

\author{
NaOto Komuro, Kichi-Suke Saito And Ryotaro TANAKA
}

Abstract. We present a new characterization of two-dimensional Banach spaces with James constant $\sqrt{2}$. As an application, we give an example of a two-dimensional Banach space with James constant $\sqrt{2}$ that is not isometrically isomorphic to any absolute, or symmetric, or $\pi / 2$-rotation invariant normed space. It is shown that this gives a counterexample to Lassak's conjecture.

Mathematics subject classification (2010): 46B20.

Keywords and phrases: James constant, isosceles orthogonality, absolute norm, symmetric norm, rotation invariant norm.

\section{REFERENCES}

[1] J. Alonso, Uniqueness properties of isosceles orthogonality in normed linear spaces, Ann. Sci. Math. Québec 18 (1994), 25-38.

[2] J. Alonso, H. MARTINI AND S. Wu, On Birkhoff orthogonality and isosceles orthogonality in normed linear spaces, Aequationes Math. 83 (2012), 153-189.

[3] G. BIRKHOFF, Orthogonality in linear metric spaces, Duke Math. J., 1 (1935), 169-172.

[4] F. F. Bonsall and J. Duncan, Numerical ranges II, Cambridge University Press, Cambridge, 1973.

[5] J. GAO AND K.-S. LAU, On the geometry of spheres in normed linear spaces, J. Aust. Math. Soc. Ser. A 48 (1990), 101-112.

[6] H. HADWIGER, Ungelöste probleme, no. 20, Elem. Math. 12 (1957), 121.

[7] C. He, H. Martini And S. Wu, On covering functionals of convex bodies, J. Math. Anal. Appl. 437 (2016), 1236-1256.

[8] D. JI, J. LI AND S. WU, On the uniqueness of isosceles orthogonality in normed linear spaces, Results Math. 59 (2011), 157-162.

[9] N. Komuro, K.-S. SAITO AND R. TANAKA, On the class of Banach spaces with James constant $\sqrt{2}$, Math. Nachr. 289 (2016), 1005-1020.

[10] N. Komuro, K.-S. SAito And R. TANAKA, On the class of Banach spaces with James constant $\sqrt{2}$, II, Mediterr. J. Math. 13 (2016), 4039-4061.

[11] M. LASSAK, Covering a plane convex body by four homothetical copies with the smallest positive ratio, Geom. Dedicata 21 (1986), 157-167.

[12] B. D. RoberTs, On the geometry of abstract vector spaces, Tohoku Math. J. 39 (1934), 42-59. 